\title{
Modified $\psi$-contractive mappings in ordered metric spaces and applications
}

\author{
Hemant Kumar Nashine' ${ }^{1}$ Zoran Golubović ${ }^{2}$ and Zoran Kadelburg ${ }^{3 *}$
}

\author{
"Correspondence: \\ kadelbur@matf.bg.ac.rs \\ ${ }^{3}$ Faculty of Mathematics, University \\ of Belgrade, Studentski trg 16, \\ Beograd, 11000, Serbia \\ Full list of author information is \\ available at the end of the article
}

\begin{abstract}
We set up two new variants of $\psi$-contractive mappings designed for two and three maps in metric spaces and originate common fixed point theorems for $\mathcal{T}$-strictly weakly isotone increasing mappings and relatively weakly increasing mappings in complete ordered metric spaces. To demonstrate our results, we give some examples throughout the paper. At the same time, as applications of the presented theorems, we get hold of common fixed point results for generalized contractions of integral type and we prove an existence theorem for solutions of a system of integral equations.
\end{abstract}

MSC: $47 \mathrm{H} 10 ; 45 F 05$

Keywords: partially ordered set; asymptotically regular map; orbitally complete metric space; orbital continuity; weakly increasing maps; $\mathcal{T}$-strictly weakly isotone increasing mappings

\section{Introduction and preliminaries}

The celebrated Banach's contraction mapping principle [1] is one of the cornerstones in the development of nonlinear analysis. Fixed point theorems have applications not only in the various branches of mathematics, but also in economics, chemistry, biology, computer science, engineering, and other areas. In particular, such theorems are used to demonstrate the existence and uniqueness of solutions of differential equations, integral equations, functional equations, partial differential equations and others. Owing to the magnitude, the generalizations of the Banach fixed point theorem have been explored heavily by many authors (see, e.g., [2] and references cited therein).

Browder and Petryshyn [3] introduced the concept of orbital continuity as well as of asymptotic regularity of a self-map at a point in a metric space. Cirić [4] introduced the concept of an orbitally complete metric space. Sastry et al. [5] extended these concepts to two and three mappings and employed them to prove common fixed point results for commuting mappings. In what follows, we collect such definitions for three maps.

Definition 1 Let $\mathcal{S}, \mathcal{T}, \mathcal{R}$ be three self-mappings defined on a metric space $(\mathcal{X}, d)$.

1. If for a point $x_{0} \in \mathcal{X}$, there exits a sequence $\left\{x_{n}\right\}$ in $\mathcal{X}$ such that $\mathcal{R} x_{2 n+1}=\mathcal{S} x_{2 n}$, $\mathcal{R} x_{2 n+2}=\mathcal{T} x_{2 n+1}, n=0,1,2, \ldots$, then the set $\mathcal{O}\left(x_{0} ; \mathcal{S}, \mathcal{T}, \mathcal{R}\right)=\left\{\mathcal{R} x_{n}: n=1,2, \ldots\right\}$ is called the orbit of $(\mathcal{S}, \mathcal{T}, \mathcal{R})$ at $x_{0}$.

2. The space $(\mathcal{X}, d)$ is said to be $(\mathcal{S}, \mathcal{T}, \mathcal{R})$-orbitally complete at $x_{0}$ if every Cauchy sequence in $\mathcal{O}\left(x_{0} ; \mathcal{S}, \mathcal{T}, \mathcal{R}\right)$ converges in $\mathcal{X}$.

C 2012 Nashine et al : licensee Springer. This is an Open Access article distributed under the terms of the Creative Commons Attribution License (http://creativecommons.org/licenses/by/2.0), which permits unrestricted use, distribution, and reproduction in any medium, provided the original work is properly cited. 
3. The map $\mathcal{R}$ is said to be orbitally continuous at $x_{0}$ if it is continuous on $\mathcal{O}\left(x_{0} ; \mathcal{S}, \mathcal{T}, \mathcal{R}\right)$

4. The pair $(\mathcal{S}, \mathcal{T})$ is said to be asymptotically regular (in short a.r.) with respect to $\mathcal{R}$ at $x_{0}$ if there exists a sequence $\left\{x_{n}\right\}$ in $\mathcal{X}$ such that $\mathcal{R} x_{2 n+1}=\mathcal{S} x_{2 n}, \mathcal{R} x_{2 n+2}=\mathcal{T} x_{2 n+1}$, $n=0,1,2, \ldots$, and $d\left(\mathcal{R} x_{n}, \mathcal{R} x_{n+1}\right) \rightarrow 0$ as $n \rightarrow \infty$.

5. If $\mathcal{R}$ is the identity mapping on $\mathcal{X}$, we omit ' $\mathcal{R}$ ' in respective definitions.

On the other hand, fixed point theory has developed rapidly in metric spaces endowed with a partial ordering. The foremost result in this direction was given by Ran and Reurings [6] who presented its applications to matrix equations. Subsequently, Nieto and Rodríguez-López [7] extended this result for nondecreasing mappings and applied it to obtain a unique solution for a first-order ordinary differential equation with periodic boundary conditions. From then on, a number of authors have obtained many fixed point theorems in ordered metric spaces. For more details, see, e.g., [8-12] and the references cited therein. Many good quality works have been produced by the authors like Aydi, Karapinar, and Shatanawi in this area. Very recently, Chen [13] introduced $\psi$-contractive mappings (see Definition 5) and proved some fixed point theorems in an ordered metric space, thus extending and improving the results given in [14]. One of the main results in [13] is the following theorem.

Theorem 1 Let $(\mathcal{X}, d, \preceq)$ be a complete partially ordered metric space such that for each nondecreasing sequence $\left\{x_{n}\right\}$ in $\mathcal{X}$ converging to $z \in \mathcal{X}, x_{n} \preceq z$ holds for each $n \in \mathbb{N}$. Suppose that $\mathcal{T}: \mathcal{X} \rightarrow \mathcal{X}$ is a nondecreasing $\psi$-contractive self-map. If there exists an $x_{0} \in \mathcal{X}$ such that $x_{0} \preceq \mathcal{T} x_{0}$, then $\mathcal{T}$ has a fixed point in $\mathcal{X}$.

In this paper, we extend the results of Chen [13] (and hence some other related common fixed point results) in two directions. The first is treated in Section 3, where the notion of a $\psi_{\mathcal{S}}$-contractive condition is introduced in metric spaces. The existence and (under additional assumptions) uniqueness of common fixed points is obtained under the assumptions that respective mappings are strictly weakly isotone increasing and that they satisfy the $\psi_{\mathcal{S}}$-contractive condition. In Section 4, we consider the case of three self-mappings $\mathcal{S}, \mathcal{T}, \mathcal{R}$ where the pair $(\mathcal{S}, \mathcal{T})$ is $\mathcal{R}$-relatively asymptotically regular and relatively weakly increasing, while the new contractive condition, named $\psi_{\mathcal{S}, \mathcal{R}}$-contraction, is established.

We supply appropriate examples to make the validity of the propositions of our results obvious. To end with, as applications of the presented theorems, we achieve common fixed point results for generalized contractions of integral type and we prove the existence theorem for solutions of a system of integral equations.

\section{Notation and definitions}

All the way through this paper, by $\mathbb{R}^{+}$we designate the set of nonnegative real numbers, while $\mathbb{N}$ is the set of natural numbers and $\mathbb{N}_{0}=\mathbb{N} \cup\{0\}$.

First, we introduce some further notation and definitions that will be used later.

If $(\mathcal{X}, \preceq)$ is a partially ordered set, then $x, y \in \mathcal{X}$ are called comparable if $x \preceq y$ or $y \preceq x$ holds. A subset $\mathcal{K}$ of $\mathcal{X}$ is said to be totally ordered if every two elements of $\mathcal{K}$ are comparable. If $\mathcal{T}: \mathcal{X} \rightarrow \mathcal{X}$ is such that, for $x, y \in \mathcal{X}, x \preceq y$ implies $\mathcal{T} x \preceq \mathcal{T} y$, then the mapping $\mathcal{T}$ is said to be nondecreasing. 
Definition 2 Let $(\mathcal{X}, \preceq)$ be a partially ordered set and $\mathcal{S}, \mathcal{T}: \mathcal{X} \rightarrow \mathcal{X}$.

1. $[15,16]$ The pair $(\mathcal{S}, \mathcal{T})$ is called weakly increasing if $\mathcal{S} x \preceq \mathcal{T} \mathcal{S} x$ and $\mathcal{T} x \preceq \mathcal{S T} x$ for all $x \in \mathcal{X}$

2. [15-17] The mapping $\mathcal{S}$ is said to be $\mathcal{T}$-weakly isotone increasing if for all $x \in \mathcal{X}$, we have $\mathcal{S} x \preceq \mathcal{T} \mathcal{S} x \preceq \mathcal{S} \mathcal{T} \mathcal{S} x$.

3. [18] The mapping $\mathcal{S}$ is said to be $\mathcal{T}$-strictly weakly isotone increasing if for all $x \in \mathcal{X}$ such that $x \prec \mathcal{S} x$, we have $\mathcal{S} x \prec \mathcal{T} \mathcal{S} x \prec \mathcal{S T} \mathcal{S} x$.

4. [11] Let $\mathcal{R}: \mathcal{X} \rightarrow \mathcal{X}$ be such that $\mathcal{T} \mathcal{X} \subseteq \mathcal{R} \mathcal{X}$ and $\mathcal{S} \mathcal{X} \subseteq \mathcal{R} \mathcal{X}$, and denote $\mathcal{R}^{-1}(x):=\{u \in \mathcal{X}: \mathcal{R} u=x\}$ for $x \in \mathcal{X}$. We say that $\mathcal{T}$ and $\mathcal{S}$ are weakly increasing with respect to $\mathcal{R}$ if for all $x \in \mathcal{X}$, we have

$$
\mathcal{T} x \preceq \mathcal{S} y, \quad \forall y \in \mathcal{R}^{-1}(\mathcal{T} x) \quad \text { and } \quad \mathcal{S} x \preceq \mathcal{T} y, \quad \forall y \in \mathcal{R}^{-1}(\mathcal{S} x)
$$

5. [19] The mapping $\mathcal{T}$ is called dominating if $x \preceq \mathcal{T} x$ for each $x$ in $\mathcal{X}$.

Remark 1 (1) None of two weakly increasing mappings need to be nondecreasing. There exist some examples to illustrate this fact in [20].

(2) If $\mathcal{S}, \mathcal{T}: \mathcal{X} \rightarrow \mathcal{X}$ are weakly increasing, then $\mathcal{S}$ is $\mathcal{T}$-weakly isotone increasing and hence $\mathcal{S}$ can be $\mathcal{T}$-strictly weakly isotone increasing.

(3) $\mathcal{S}$ can be $\mathcal{T}$-strictly weakly isotone increasing, while some of these two mappings can be not strictly increasing (see the following example).

(4) If $\mathcal{R}$ is the identity mapping $(\mathcal{R} x=x$ for all $x \in \mathcal{X})$, then $\mathcal{T}$ and $\mathcal{S}$ are weakly increasing with respect to $\mathcal{R}$ if and only if they are weakly increasing mappings.

Example 1 Let $\mathcal{X}=[0,+\infty)$ be endowed with the usual ordering and define $\mathcal{S}, \mathcal{T}: \mathcal{X} \rightarrow \mathcal{X}$ as

$$
\mathcal{S} x=\left\{\begin{array}{ll}
2 x, & \text { if } x \in[0,1], \\
3 x, & \text { if } x>1 ;
\end{array} \quad \mathcal{T} x= \begin{cases}2, & \text { if } x \in[0,1] \\
2 x, & \text { if } x>1\end{cases}\right.
$$

Clearly, we have $x \prec \mathcal{S} x \prec \mathcal{T} \mathcal{S} x \prec \mathcal{S T} \mathcal{S} x$ for all $x \in \mathcal{X}$, and so, $\mathcal{S}$ is $\mathcal{T}$-strictly weakly isotone increasing; $\mathcal{T}$ is not strictly increasing.

Definition $3([21,22])$ Let $(\mathcal{X}, d)$ be a metric space and $f, g: \mathcal{X} \rightarrow \mathcal{X}$. The mappings $f$ and $g$ are said to be compatible if $\lim _{n \rightarrow \infty} d\left(f g x_{n}, g f x_{n}\right)=0$, whenever $\left\{x_{n}\right\}$ is a sequence in $\mathcal{X}$ such that $\lim _{n \rightarrow \infty} f x_{n}=\lim _{n \rightarrow \infty} g x_{n}=t$ for some $t \in \mathcal{X}$.

Definition 4 Let $\mathcal{X}$ be a nonempty set. Then $(\mathcal{X}, d, \preceq)$ is called an ordered metric space if

(i) $(\mathcal{X}, d)$ is a metric space,

(ii) $(\mathcal{X}, \preceq)$ is a partially ordered set.

The space $(\mathcal{X}, d, \preceq)$ is called regular if the following hypothesis holds: if $\left\{z_{n}\right\}$ is a nondecreasing sequence in $\mathcal{X}$ with respect to $\preceq$ such that $z_{n} \rightarrow z \in \mathcal{X}$ as $n \rightarrow \infty$, then $z_{n} \preceq z$.

\section{Common fixed points for generalized $\psi_{\mathcal{S}}$-contractive mappings}

Inspired by the notion of a $\psi$-contractive mapping given in [13], we first introduce the notion of $\psi_{\mathcal{S}}$-contractive mappings in metric spaces. 
For convenience, we denote by $\mathbf{F}$ the class of functions $\psi: \mathbb{R}^{+^{5}} \rightarrow \mathbb{R}^{+}$satisfying the following conditions:

$\left(C_{1}\right) \psi$ is a strictly increasing and continuous function in each coordinate, and

$\left(C_{2}\right)$ for all $t \in \mathbb{R}^{+} \backslash\{0\}, \psi(t, t, t, 0,2 t)<t, \psi(t, t, t, 2 t, 0)<t, \psi(0,0, t, t, 0)<t, \psi(0, t, 0$, $0, t)<t$, and $\psi(t, 0,0, t, t)<t$.

The following are some easy examples of functions from class $\mathbf{F}$ :

$$
\begin{aligned}
& \psi\left(t_{1}, t_{2}, t_{3}, t_{4}, t_{5}\right)=k \max \left\{t_{1}, t_{2}, t_{3}, \frac{t_{4}}{2}, \frac{t_{5}}{2}\right\}, \quad \text { for } k \in(0,1) \\
& \psi\left(t_{1}, t_{2}, t_{3}, t_{4}, t_{5}\right)=k \max \left\{t_{1}, t_{2}, t_{3}, \frac{t_{4}+t_{5}}{2}\right\}, \quad \text { for } k \in(0,1) \\
& \psi\left(t_{1}, t_{2}, t_{3}, t_{4}, t_{5}\right)=A t_{1}+B t_{2}+C t_{3}+D \frac{t_{4}+t_{5}}{2}, \quad \text { for } A, B, C, D \geq 0, A+B+C+D<1 .
\end{aligned}
$$

Definition 5 Let $(\mathcal{X}, d, \preceq)$ be an ordered metric space, and let $\mathcal{T}, \mathcal{S}: \mathcal{X} \rightarrow \mathcal{X}$. The mappings $\mathcal{T}, \mathcal{S}$ are said to be $\psi_{\mathcal{S}}$-contractive if

$$
d(\mathcal{T} x, \mathcal{S} y) \leq \psi(d(x, y), d(x, \mathcal{T} x), d(y, \mathcal{S} y), d(x, \mathcal{S} y), d(y, \mathcal{T} x)) \quad \text { for } x \succeq y
$$

where $\psi \in \mathbf{F}$.

For $\mathcal{S}=\mathcal{T}$, this definition reduces to the definition of a $\psi$-contractive mapping from [13]. It is easy to acquire the following examples of $\psi_{\mathcal{S}}$-contractive mappings.

Example 2 Let $\mathcal{X}=\mathbb{R}^{+}$be endowed with usual metric and ordering, and let $\psi: \mathbb{R}^{+^{5}} \rightarrow \mathbb{R}^{+}$ be given by

$$
\psi\left(t_{1}, t_{2}, t_{3}, t_{4}, t_{5}\right)=\frac{3}{4} \max \left\{t_{1}, t_{2}, t_{3}, \frac{t_{4}+t_{5}}{2}\right\} .
$$

If $\mathcal{T}, \mathcal{S}: \mathcal{X} \rightarrow \mathcal{X}$ are defined by $\mathcal{T} x=\frac{1}{3} x$ and $\mathcal{S} x=\frac{1}{2} x$, then $\mathcal{T}, \mathcal{S}$ are $\psi_{\mathcal{S}}$-contractive mappings.

Example 3 Let $\mathcal{X}=\mathbb{R}^{+} \times \mathbb{R}^{+}$be endowed with the coordinate ordering (i.e., $\left(x_{1}, y_{1}\right) \leq$ $\left(x_{2}, y_{2}\right) \Leftrightarrow x_{1} \leq x_{2}$ and $\left.y_{1} \leq y_{2}\right)$ and with the metric $d: \mathcal{X} \times \mathcal{X} \rightarrow \mathbb{R}^{+}$given by

$$
d(x, y)=\left|x_{1}-y_{1}\right|+\left|x_{2}-y_{2}\right|, \quad \text { for } x=\left(x_{1}, x_{2}\right), y=\left(y_{1}, y_{2}\right) \in \mathcal{X} .
$$

Let $\psi: \mathbb{R}^{+^{5}} \rightarrow \mathbb{R}^{+}$be given by

$$
\psi\left(t_{1}, t_{2}, t_{3}, t_{4}, t_{5}\right)=\frac{3}{4} \max \left\{t_{1}, t_{2}, t_{3}, \frac{t_{4}}{2}, \frac{t_{5}}{2}\right\},
$$

and $\mathcal{T}, \mathcal{S}: \mathcal{X} \rightarrow \mathcal{X}$ be given by

$$
\mathcal{T}(x, y)=\left(\frac{1}{3} x, \frac{1}{3} y\right) \quad \text { and } \quad \mathcal{S}(x, y)=\left(\frac{1}{2} x, \frac{1}{2} y\right)
$$

Then $\mathcal{T}, \mathcal{S}$ are $\psi_{\mathcal{S}}$-contractive mappings. 
Now, we state and prove our first result.

Theorem 2 Let $(\mathcal{X}, d, \preceq)$ be a complete ordered metric space. Suppose that $\mathcal{T}, \mathcal{S}: \mathcal{X} \rightarrow \mathcal{X}$ are two mappings satisfying the $\psi_{\mathcal{S}}$-contractive condition (3.1) for all comparable $\mathcal{x}, y \in \mathcal{X}$.

We assume the following hypotheses:

(i) $\mathcal{S}$ is $\mathcal{T}$-strictly weakly isotone increasing;

(ii) there exists an $x_{0} \in \mathcal{X}$ such that $x_{0} \prec \mathcal{S} x_{0}$;

(iii) $\mathcal{S}$ or $\mathcal{T}$ is continuous at $x_{0}$.

Then $\mathcal{S}$ and $\mathcal{T}$ have a common fixed point. Moreover, the set of common fixed points of $\mathcal{S}$, $\mathcal{T}$ is totally ordered if and only if $\mathcal{S}$ and $\mathcal{T}$ have one and only one common fixed point.

Proof First of all, we show that if $\mathcal{S}$ or $\mathcal{T}$ has a fixed point, then it is a common fixed point of $\mathcal{S}$ and $\mathcal{T}$. Indeed, let $z$ be a fixed point of $\mathcal{S}$. Assume that $d(z, \mathcal{T} z)>0$. If we use the inequality (3.1), for $x=y=z$, we have

$$
\begin{aligned}
d(\mathcal{T} z, z) & =d(\mathcal{T} z, \mathcal{S} z) \leq \psi(d(z, z), d(z, \mathcal{T} z), d(z, \mathcal{S} z), d(z, \mathcal{S} z), d(z, \mathcal{T} z)) \\
& =\psi(0, d(z, \mathcal{T} z), 0,0, d(z, \mathcal{T} z))<d(z, \mathcal{T} z)
\end{aligned}
$$

which is a contradiction. Thus, $d(z, \mathcal{T} z)=0$ and so $z$ is a common fixed point of $\mathcal{S}$ and $\mathcal{T}$. Analogously, one can observe that if $z$ is a fixed point of $\mathcal{T}$, then it is a common fixed point of $\mathcal{S}$ and $\mathcal{T}$.

Let $x_{0}$ be such that $x_{0} \prec \mathcal{S} x_{0}$. We can define a sequence $\left\{x_{n}\right\}$ in $\mathcal{X}$ as follows:

$$
x_{2 n+1}=\mathcal{S} x_{2 n} \quad \text { and } \quad x_{2 n+2}=\mathcal{T} x_{2 n+1} \quad \text { for } n \in\{0,1, \ldots\} \text {. }
$$

Since $\mathcal{S}$ is $\mathcal{T}$-strictly weakly isotone increasing, we have

$$
\begin{aligned}
& x_{1}=\mathcal{S} x_{0} \prec \mathcal{T S} x_{0}=\mathcal{T} x_{1}=x_{2} \prec \mathcal{S} \mathcal{S} x_{0}=\mathcal{S} \mathcal{T} x_{1}=\mathcal{S} x_{2}=x_{3}, \\
& x_{3}=\mathcal{S} x_{2} \prec \mathcal{T} \mathcal{S} x_{2}=\mathcal{T} x_{3}=x_{4} \prec \mathcal{S} \mathcal{T} x_{2}=\mathcal{S} \mathcal{T} x_{3}=\mathcal{S} x_{4}=x_{5},
\end{aligned}
$$

and continuing this process, we get

$$
x_{1} \prec x_{2} \prec \cdots \prec x_{n} \prec x_{n+1} \prec \cdots .
$$

Now, we claim that for all $n \in \mathbb{N}$, we have

$$
d\left(x_{n+1}, x_{n+2}\right)<d\left(x_{n}, x_{n+1}\right) .
$$

Suppose to the contrary that, e.g., $d\left(x_{2 n}, x_{2 n+1}\right) \leq d\left(x_{2 n+1}, x_{2 n+2}\right)$ for some $n \in \mathbb{N}$. From (3.2) we have that $x_{2 n} \prec x_{2 n+1}$ for all $n \in \mathbb{N}$. Then from (3.1) with $x=x_{2 n+1}$ and $y=x_{2 n}$, we get

$$
\begin{aligned}
d\left(x_{2 n+2}, x_{2 n+1}\right)= & d\left(\mathcal{T} x_{2 n+1}, \mathcal{S} x_{2 n}\right) \\
\leq & \psi\left(d\left(x_{2 n+1}, x_{2 n}\right), d\left(x_{2 n+1}, \mathcal{T} x_{2 n+1}\right), d\left(x_{2 n}, \mathcal{S} x_{2 n}\right)\right. \\
& \left.d\left(x_{2 n+1}, \mathcal{S} x_{2 n}\right), d\left(x_{2 n}, \mathcal{T} x_{2 n+1}\right)\right)
\end{aligned}
$$




$$
\begin{aligned}
= & \psi\left(d\left(x_{2 n+1}, x_{2 n}\right), d\left(x_{2 n+1}, x_{2 n+2}\right), d\left(x_{2 n}, x_{2 n+1}\right),\right. \\
& \left.d\left(x_{2 n+1}, x_{2 n+1}\right), d\left(x_{2 n}, x_{2 n+2}\right)\right) \\
\leq & \psi\left(d\left(x_{2 n+1}, x_{2 n}\right), d\left(x_{2 n+1}, x_{2 n+2}\right), d\left(x_{2 n}, x_{2 n+1}\right),\right. \\
& \left.0, d\left(x_{2 n}, x_{2 n+1}\right)+d\left(x_{2 n+1}, x_{2 n+2}\right)\right) \\
\leq & \psi\left(d\left(x_{2 n+1}, x_{2 n+2}\right), d\left(x_{2 n+1}, x_{2 n+2}\right), d\left(x_{2 n+1}, x_{2 n+2}\right), 0,2 d\left(x_{2 n+1}, x_{2 n+2}\right)\right) \\
< & d\left(x_{2 n+1}, x_{2 n+2}\right),
\end{aligned}
$$

a contradiction. Hence, we deduce that for each $n \in \mathbb{N}$,

$$
d\left(x_{2 n+2}, x_{2 n+1}\right)<d\left(x_{2 n+1}, x_{2 n}\right) .
$$

Similarly, we can prove that $d\left(x_{2 n+1}, x_{2 n}\right)<d\left(x_{2 n}, x_{2 n-1}\right)$ for all $n \geq 1$. Therefore, we conclude that (3.3) holds.

Let us denote $c_{n}=d\left(x_{n+1}, x_{n}\right)$. Then from (3.3), $c_{n}$ is a nonincreasing sequence and bounded below. Thus, it must converge to some $c \geq 0$. If $c>0$, then similarly as above (e.g., for $n=2 k$ ), we have

$$
c_{n+1} \leq \psi\left(c_{n}, c_{n+1}, c_{n}, 0,2 c_{n}\right)
$$

Passing to the limit as $n \rightarrow \infty$, we have

$$
c \leq \psi(c, c, c, 0,2 c)<c,
$$

which is a contradiction. Hence,

$$
\lim _{n \rightarrow \infty} d\left(x_{n+1}, x_{n}\right)=0
$$

Next, we claim that $\left\{x_{n}\right\}$ is a Cauchy sequence. From (3.4), it will be sufficient to show that $\left\{x_{2 n}\right\}$ is a Cauchy sequence. We proceed with negation and suppose that $\left\{x_{2 n}\right\}$ is not Cauchy. Then we can find a $\delta>0$ such that for each even integer $2 k$, there exist even integers $2 m_{k}>2 n_{k}>2 k$ such that

$$
d\left(x_{2 n_{k}}, x_{2 m_{k}}\right) \geq \delta \quad \text { for } k \in\{1,2, \ldots\}
$$

We may also assume

$$
d\left(x_{2 n_{k}}, x_{2 m_{k}-2}\right)<\delta
$$

by choosing $2 m_{k}$ to be the smallest number exceeding $2 n_{k}$ for which (3.5) holds. Now (3.4), (3.5), and (3.6) imply

$$
\begin{aligned}
0 & <\delta \leq d\left(x_{2 n_{k}}, x_{2 m_{k}}\right) \leq d\left(x_{2 n_{k}}, x_{2 m_{k}-2}\right)+d\left(x_{2 m_{k}-2}, x_{2 m_{k}-1}\right)+d\left(x_{2 m_{k}-1}, x_{2 m_{k}}\right) \\
& <\delta+d\left(x_{2 m_{k}-2}, x_{2 m_{k}-1}\right)+d\left(x_{2 m_{k}-1}, x_{2 m_{k}}\right) \rightarrow \delta, \quad \text { as } k \rightarrow \infty,
\end{aligned}
$$


and hence

$$
\lim _{k \rightarrow \infty} d\left(x_{2 n_{k}}, x_{2 m_{k}}\right)=\delta
$$

Also, by the triangular inequality,

$$
\left|d\left(x_{2 n_{k}}, x_{2 m_{k}-1}\right)-d\left(x_{2 n_{k}}, x_{2 m_{k}}\right)\right| \leq d\left(x_{2 m_{k}-1}, x_{2 m_{k}}\right)
$$

and

$$
\begin{aligned}
& \left|d\left(x_{2 n_{k}+1}, x_{2 m_{k}-1}\right)-d\left(x_{2 n_{k}}, x_{2 m_{k}}\right)\right| \\
& \quad \leq d\left(x_{2 m_{k}-1}, x_{2 m_{k}}\right)+d\left(x_{2 n_{k}}, x_{2 n_{k}+1}\right) .
\end{aligned}
$$

Therefore, we get

$$
\lim _{k \rightarrow \infty} d\left(x_{2 n_{k}}, x_{2 m_{k}-1}\right)=\delta
$$

and

$$
\lim _{k \rightarrow \infty} d\left(x_{2 n_{k}+1}, x_{2 m_{k}-1}\right)=\delta .
$$

Since $\mathcal{T}, \mathcal{S}$ are $\psi_{\mathcal{S}}$-contractive mappings, we also have

$$
\begin{aligned}
\delta \leq & d\left(x_{2 n_{k}}, x_{2 m_{k}}\right) \\
\leq & d\left(x_{2 n_{k}}, x_{2 n_{k}+1}\right)+d\left(x_{2 n_{k}+1}, x_{2 m_{k}}\right) \\
= & d\left(x_{2 n_{k}}, x_{2 n_{k}+1}\right)+d\left(\mathcal{T} x_{2 m_{k}-1}, \mathcal{S} x_{2 n_{k}}\right) \\
\leq & d\left(x_{2 n_{k}}, x_{2 n_{k}+1}\right)+\psi\left(d\left(x_{2 m_{k}-1}, x_{2 n_{k}}\right), d\left(x_{2 m_{k}-1}, x_{2 m_{k}}\right),\right. \\
& \left.d\left(x_{2 n_{k}}, x_{2 n_{k}+1}\right), d\left(x_{2 m_{k}-1}, x_{2 n_{k}+1}\right), d\left(x_{2 m_{k}}, x_{2 n_{k}}\right)\right) .
\end{aligned}
$$

Passing to the limit as $k \rightarrow \infty$, we get

$$
\delta \leq 0+\psi(\delta, 0,0, \delta, \delta)<\delta,
$$

a contradiction. It follows that $\left\{x_{n}\right\}$ must be a Cauchy sequence.

Since $\mathcal{X}$ is complete, there exists $z \in \mathcal{X}$ such that $\lim _{n \rightarrow \infty} x_{n}=z$. Moreover, the continuity of $\mathcal{T}$ implies that

$$
z=\lim _{n \rightarrow \infty} x_{n+1}=\lim _{n \rightarrow \infty} \mathcal{T} x_{n}=\mathcal{T} z
$$

Similarly, if $\mathcal{S}$ is continuous, we have that $\mathcal{S} z=z$. Using the argument from the beginning of the proof, we conclude that $\mathcal{S}$ and $\mathcal{T}$ have a common fixed point.

Now, suppose that the set of common fixed points of $\mathcal{T}$ and $\mathcal{S}$ is totally ordered. We claim that there is a unique common fixed point of $\mathcal{T}$ and $\mathcal{S}$. Assume to the contrary that 
$\mathcal{S} u=\mathcal{T} u=u$ and $\mathcal{S} v=\mathcal{T} v=v$, but $u \neq v$. By supposition, we can replace $x$ by $u$ and $y$ by $v$ in (3.1), and by the continuity of $\psi$, we obtain

$$
\begin{aligned}
d(v, u) & =d(\mathcal{T} v, \mathcal{S} u) \leq \psi(d(v, u), d(v, \mathcal{T} v), d(u, \mathcal{S} u), d(v, \mathcal{S} u), d(u, \mathcal{T} v)) \\
& \leq \psi(d(v, u), 0,0, d(v, u), d(u, v))<d(v, u)
\end{aligned}
$$

a contradiction. Hence, $u=v$. The converse is trivial. So, we have completed the proof.

We are also able to prove the existence of a common fixed point of two mappings without using the continuity of $\mathcal{S}$ or $\mathcal{T}$. More precisely, we have the following theorem.

Theorem 3 Let $(\mathcal{X}, d, \preceq)$ and $\mathcal{S}, \mathcal{T}: \mathcal{X} \rightarrow \mathcal{X}$ satisfy all the conditions of Theorem 2, except that the condition (iii) is substituted by

(iii') $\mathcal{X}$ is regular.

Then the same conclusions as in Theorem 2 hold.

Proof Following the proof of Theorem 2, we have that $\left\{x_{n}\right\}$ is a Cauchy sequence in $(\mathcal{X}, d)$ which is complete. Then there exists $z \in \mathcal{X}$ such that

$$
\lim _{n \rightarrow \infty} x_{n}=z
$$

Now suppose that $d(z, \mathcal{S} z)>0$. From regularity of $\mathcal{X}$, we have $x_{2 n} \preceq z$ for all $n \in \mathbb{N}$. Hence, we can apply the considered contractive condition. Then setting $x=x_{2 n+1}$ and $y=z$ in (3.1), we obtain

$$
\begin{aligned}
d\left(x_{2 n+2}, \mathcal{S} z\right)= & d\left(\mathcal{T} x_{2 n+1}, \mathcal{S} z\right) \\
\leq & \psi\left(d\left(x_{2 n+1}, z\right), d\left(x_{2 n+1}, \mathcal{T} x_{2 n+1}\right), d(z, \mathcal{S} z),\right. \\
& \left.d\left(x_{2 n+1}, \mathcal{S} z\right), d\left(z, \mathcal{T} x_{2 n+1}\right)\right) \\
\leq & \psi\left(d\left(x_{2 n+1}, z\right), d\left(x_{2 n+1}, x_{2 n+2}\right), d(z, \mathcal{S} z)\right. \\
& \left.d\left(x_{2 n+1}, \mathcal{S} z\right), d\left(z, x_{2 n+2}\right)\right) .
\end{aligned}
$$

Passing to the limit as $n \rightarrow \infty$ and using the properties of $\psi$, we have

$$
d(z, \mathcal{S} z) \leq \psi(0,0, d(z, \mathcal{S} z), d(z, \mathcal{S} z), 0)<d(z, \mathcal{S} z)
$$

and this is a contradiction. Hence, $d(z, \mathcal{S} z)=0$, i.e., $z=\mathcal{S} z$. Analogously, for $x=z$ and $y=x_{2 n}$, one can prove that $\mathcal{T} z=z$. It follows that $z=\mathcal{S} z=\mathcal{T} z$, that is, $\mathcal{T}$ and $\mathcal{S}$ have a common fixed point.

Corollary 1 Let $(\mathcal{X}, d, \preceq)$ be a complete ordered metric space. Suppose $\mathcal{T}, \mathcal{S}: \mathcal{X} \rightarrow \mathcal{X}$ are two mappings satisfying the $\psi_{\mathcal{S}}$-contractive condition for all comparable $x, y \in \mathcal{X}$.

We assume the following hypotheses:

(i') $\mathcal{S}$ and $\mathcal{T}$ are weakly increasing;

(iii') $\mathcal{X}$ is regular. 
Then $\mathcal{S}$ and $\mathcal{T}$ have a common fixed point. Moreover, the set of common fixed points of $\mathcal{S}$, $\mathcal{T}$ is totally ordered if and only if $\mathcal{S}$ and $\mathcal{T}$ have one and only one common fixed point.

Putting $\mathcal{S}=\mathcal{T}$ in Theorem 2 and Theorem 3, we obtain easily the following result.

Corollary 2 Let $(\mathcal{X}, d, \preceq)$ be a complete ordered metric space. Suppose $\mathcal{T}: \mathcal{X} \rightarrow \mathcal{X}$ is a mapping satisfying the $\psi$-contractive condition for all comparable $x, y \in \mathcal{X}$. Also suppose that $\mathcal{T} x \prec \mathcal{T}(\mathcal{T} x)$ for all $x \in \mathcal{X}$ such that $x \prec \mathcal{T} x$. If there exists an $x_{0} \in \mathcal{X}$ such that $x_{0} \prec$ $\mathcal{T} x_{0}$ and the condition

\section{$\mathcal{T}$ is continuous, or $\mathcal{X}$ is regular}

holds, then $\mathcal{T}$ has a fixed point. Moreover, the set of fixed points of $\mathcal{T}$ is totally ordered if and only if it is a singleton.

We present an example showing how our results can be used.

Example 4 Let the set $\mathcal{X}=[0,+\infty)$ be equipped with the usual metric $d$ and the order defined by

$$
x \preceq y \quad \Longleftrightarrow \quad x=y \vee(x, y \in[0,1] \wedge x \geq y) .
$$

Consider the following self-mappings on $\mathcal{X}$ :

$$
\mathcal{T} x=\left\{\begin{array}{ll}
\frac{2}{3}+\frac{1}{3} x, & 0 \leq x \leq 1, \\
3 x-2, & x>1,
\end{array} \quad \mathcal{S} x= \begin{cases}\frac{1}{2}+\frac{1}{2} x, & 0 \leq x \leq 1, \\
2 x-1, & x>1\end{cases}\right.
$$

Take $\psi \in \mathbf{F}$ given by

$$
\psi\left(t_{1}, t_{2}, t_{3}, t_{4}, t_{5}\right)=\frac{5}{6} \max \left\{t_{1}, t_{2}, t_{3}, \frac{t_{4}+t_{5}}{2}\right\} .
$$

Then it is easy to show that all the conditions of Theorem 2 are fulfilled. The contractive condition (3.1) is trivially satisfied if $x=y$. Suppose that $x \succeq y$ and $0 \leq x, y \leq 1$, i.e., $x \leq y$. Then (3.1) takes the form

$$
\begin{aligned}
& \left|\frac{1}{6}+\frac{1}{3} x-\frac{1}{2} y\right| \\
& \quad \leq \frac{5}{6} \max \left\{|x-y|, \frac{2}{3}|x-1|, \frac{1}{2}|y-1|, \frac{1}{2}\left[\left|x-\frac{1}{2}-\frac{1}{2} y\right|+\left|y-\frac{2}{3}-\frac{1}{3} x\right|\right]\right\} .
\end{aligned}
$$

Using substitution $x=1-\xi, y=1-\xi t, 0 \leq \xi \leq 1, t \geq 0$, the last inequality reduces to

$$
|3 t-2| \leq 5 \max \left\{|1-t|, \frac{2}{3}, \frac{1}{2} t, \frac{1}{2}\left[\left|\frac{1}{2} t-1\right|+\left|t-\frac{1}{3}\right|\right]\right\},
$$

and can be checked by discussion on possible values for $t \geq 0$. Hence, all the conditions of Theorem 2 are satisfied and $\mathcal{S}, \mathcal{T}$ have a common fixed point (which is $z=1$ ). 


\section{Common fixed points for $\psi_{\mathcal{S}, \mathcal{R}}$-contractive mappings}

In this section, we generalize and improve the results of Section 3 (hence results given in [13]) for three maps (under additional conditions).

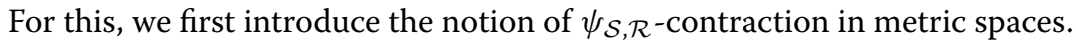

Definition 6 Let $(\mathcal{X}, d)$ be a metric space. Mappings $\mathcal{T}, \mathcal{S}, \mathcal{R}: \mathcal{X} \rightarrow \mathcal{X}$ are called $\psi_{\mathcal{S}, \mathcal{R}^{-}}$ contractive if

$$
d(\mathcal{T} x, \mathcal{S} y) \leq \psi(d(\mathcal{R} x, \mathcal{R} y), d(\mathcal{R} x, \mathcal{T} x), d(\mathcal{R} y, \mathcal{S} y), d(\mathcal{R} x, \mathcal{S} y), d(\mathcal{R} y, \mathcal{T} x))
$$

for $\mathcal{R} x \geq \mathcal{R} y$, where $\psi \in \mathbf{F}$.

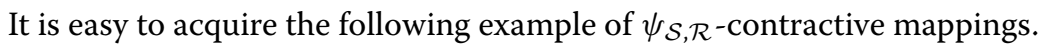

Example 5 Let $\mathcal{X}=\mathbb{R}^{+}$be endowed with standard metric and order. Let $\psi: \mathbb{R}^{+5} \rightarrow \mathbb{R}^{+}$be given by

$$
\psi\left(t_{1}, t_{2}, t_{3}, t_{4}, t_{5}\right)=\frac{3}{4} \max \left\{t_{1}, t_{2}, t_{3}, \frac{t_{4}}{2}, \frac{t_{5}}{2}\right\},
$$

and let $\mathcal{T}, \mathcal{S}, \mathcal{R}: \mathcal{X} \rightarrow \mathcal{X}$ be defined as

$$
\mathcal{T} x=\frac{1}{5} x, \quad \mathcal{S} x=\frac{1}{2} x \quad \text { and } \quad \mathcal{R} x=10 x .
$$

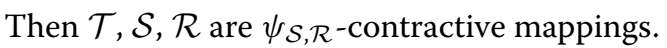

Now, we state and prove our second main result.

Theorem 4 Let $(\mathcal{X}, d, \preceq)$ be an ordered metric space, and let $\mathcal{T}, \mathcal{S}$, and $\mathcal{R}$ be self-maps

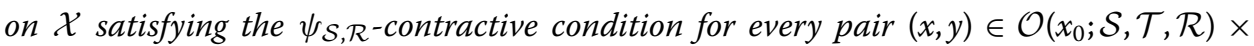
$\mathcal{O}\left(x_{0} ; \mathcal{S}, \mathcal{T}, \mathcal{R}\right)$ (for some $\left.x_{0}\right)$ such that $\mathcal{R} x$ and $\mathcal{R} y$ are comparable.

We assume the following hypotheses:

(i) $(\mathcal{S}, \mathcal{T})$ is a.r. with respect to $\mathcal{R}$ at $x_{0} \in \mathcal{X}$;

(ii) $\mathcal{X}$ is $(\mathcal{S}, \mathcal{T}, \mathcal{R})$-orbitally complete at $x_{0}$;

(iii) $\mathcal{T}$ and $\mathcal{S}$ are weakly increasing with respect to $\mathcal{R}$;

(iv) $\mathcal{T}$ and $\mathcal{S}$ are dominating maps.

Assume either

(a) $\mathcal{S}$ and $\mathcal{R}$ are compatible; $\mathcal{S}$ or $\mathcal{R}$ is orbitally continuous at $x_{0}$ or

(b) $\mathcal{T}$ and $\mathcal{R}$ are compatible; $\mathcal{T}$ or $\mathcal{R}$ is orbitally continuous at $x_{0}$.

Then $\mathcal{S}, \mathcal{T}$, and $\mathcal{R}$ have a common fixed point. Moreover, the set of common fixed points of $\mathcal{S}, \mathcal{T}$, and $\mathcal{R}$ is well ordered if and only if $\mathcal{S}, \mathcal{T}$, and $\mathcal{R}$ have one and only one common fixed point.

Proof Since $(\mathcal{S}, \mathcal{T})$ is a.r. with respect to $\mathcal{R}$ at $x_{0}$ in $\mathcal{X}$, there exists a sequence $\left\{x_{n}\right\}$ in $\mathcal{X}$ such that

$$
\mathcal{R} x_{2 n+1}=\mathcal{S} x_{2 n}, \quad \mathcal{R} x_{2 n+2}=\mathcal{T} x_{2 n+1}, \quad \forall n \in \mathbb{N}_{0},
$$


and

$$
\lim _{n \rightarrow \infty} d\left(\mathcal{R} x_{n}, \mathcal{R} x_{n+1}\right)=0
$$

holds. We claim that

$$
\mathcal{R} x_{n} \preceq \mathcal{R} x_{n+1}, \quad \forall n \in \mathbb{N}_{0}
$$

To this aim, we will use the increasing property with respect to $\mathcal{R}$ satisfied by the mappings $\mathcal{T}$ and $\mathcal{S}$. From (4.2), we have

$$
\mathcal{R} x_{1}=\mathcal{S} x_{0} \preceq \mathcal{T} y, \quad \forall y \in \mathcal{R}^{-1}\left(\mathcal{S} x_{0}\right) .
$$

Since $\mathcal{R} x_{1}=\mathcal{S} x_{0}, x_{1} \in \mathcal{R}^{-1}\left(\mathcal{S} x_{0}\right)$ and we get

$$
\mathcal{R} x_{1}=\mathcal{S} x_{0} \preceq \mathcal{T} x_{1}=\mathcal{R} x_{2}
$$

Again,

$$
\mathcal{R} x_{2}=\mathcal{T} x_{1} \preceq \mathcal{S} y, \quad \forall y \in \mathcal{R}^{-1}\left(\mathcal{T} x_{1}\right) .
$$

Since $x_{2} \in \mathcal{R}^{-1}\left(\mathcal{T} x_{1}\right)$, we get

$$
\mathcal{R} x_{2}=\mathcal{T} x_{1} \preceq \mathcal{S} x_{2}=\mathcal{R} x_{3} .
$$

Hence, by induction, (4.4) holds. Therefore, we can apply (4.1) for $x=x_{p}$ and $y=x_{q}$ for all $p$ and $q$.

Next, we claim that $\left\{\mathcal{R} x_{n}\right\}$ is a Cauchy sequence. From (4.3), it will be sufficient to show that $\left\{\mathcal{R} x_{2 n}\right\}$ is a Cauchy sequence. We proceed with negation and suppose that $\left\{\mathcal{R} x_{2 n}\right\}$ is not Cauchy. Then we can find a $\delta>0$ such that for each even integer $2 k$, there exist even integers $2 m_{k}>2 n_{k}>2 k$ such that

$$
d\left(\mathcal{R} x_{2 n_{k}}, \mathcal{R} x_{2 m_{k}}\right) \geq \delta \quad \text { for } k \in\{1,2, \ldots\}
$$

We may also assume

$$
d\left(\mathcal{R} x_{2 n_{k}}, \mathcal{R} x_{2 m_{k}-2}\right)<\delta
$$

by choosing $2 m_{k}$ to be the smallest number exceeding $2 n_{k}$ for which (4.5) holds. Now (4.3), (4.5), and (4.6) imply

$$
\begin{aligned}
0 & <\delta \leq d\left(\mathcal{R} x_{2 n_{k}}, \mathcal{R} x_{2 m_{k}}\right) \\
& \leq d\left(\mathcal{R} x_{2 n_{k}}, \mathcal{R} x_{2 m_{k}-2}\right)+d\left(\mathcal{R} x_{2 m_{k}-2}, \mathcal{R} x_{2 m_{k}-1}\right)+d\left(\mathcal{R} x_{2 m_{k}-1}, \mathcal{R} x_{2 m_{k}}\right) \\
& <\delta+d\left(\mathcal{R} x_{2 m_{k}-2}, \mathcal{R} x_{2 m_{k}-1}\right)+d\left(\mathcal{R} x_{2 m_{k}-1}, \mathcal{R} x_{2 m_{k}}\right) \rightarrow \delta, \quad \text { as } k \rightarrow \infty
\end{aligned}
$$


and hence

$$
\lim _{k \rightarrow \infty} d\left(\mathcal{R} x_{2 n_{k}}, \mathcal{R} x_{2 m_{k}}\right)=\delta
$$

Also, by the triangular inequality,

$$
\left|d\left(\mathcal{R} x_{2 n_{k}}, \mathcal{R} x_{2 m_{k}-1}\right)-d\left(\mathcal{R} x_{2 n_{k}}, \mathcal{R} x_{2 m_{k}}\right)\right| \leq d\left(\mathcal{R} x_{2 m_{k}-1}, \mathcal{R} x_{2 m_{k}}\right)
$$

and

$$
\begin{aligned}
& \left|d\left(\mathcal{R} x_{2 n_{k}+1}, \mathcal{R} x_{2 m_{k}-1}\right)-d\left(\mathcal{R} x_{2 n_{k}}, \mathcal{R} x_{2 m_{k}}\right)\right| \\
& \quad \leq d\left(\mathcal{R} x_{2 m_{k}-1}, \mathcal{R} x_{2 m_{k}}\right)+d\left(\mathcal{R} x_{2 n_{k}}, \mathcal{R} x_{2 n_{k}+1}\right) .
\end{aligned}
$$

Therefore, we get

$$
\lim _{k \rightarrow \infty} d\left(\mathcal{R} x_{2 n_{k}}, \mathcal{R} x_{2 m_{k}-1}\right)=\delta
$$

and

$$
\lim _{k \rightarrow \infty} d\left(\mathcal{R} x_{2 n_{k}+1}, \mathcal{R} x_{2 m_{k}-1}\right)=\delta .
$$

Since $\mathcal{T}, \mathcal{S}, \mathcal{R}$ are $\psi_{\mathcal{S}, \mathcal{R}}$-contractive mappings, we also have

$$
\begin{aligned}
\delta \leq & d\left(\mathcal{R} x_{2 n_{k}}, \mathcal{R} x_{2 m_{k}}\right) \leq d\left(\mathcal{R} x_{2 n_{k}}, \mathcal{R} x_{2 n_{k}+1}\right)+d\left(\mathcal{R} x_{2 n_{k}+1}, \mathcal{R} x_{2 m_{k}}\right) \\
= & d\left(\mathcal{R} x_{2 n_{k}}, \mathcal{R} x_{2 n_{k}+1}\right)+d\left(\mathcal{T} x_{2 m_{k}-1}, \mathcal{S} x_{2 n_{k}}\right) \\
\leq & d\left(\mathcal{R} x_{2 n_{k}}, \mathcal{R} x_{2 n_{k}+1}\right)+\psi\left(d\left(\mathcal{R} x_{2 m_{k}-1}, \mathcal{R} x_{2 n_{k}}\right), d\left(\mathcal{R} x_{2 m_{k}-1}, \mathcal{R} x_{2 m_{k}}\right),\right. \\
& \left.d\left(\mathcal{R} x_{2 n_{k}}, \mathcal{R} x_{2 n_{k}+1}\right), d\left(\mathcal{R} x_{2 m_{k}-1}, \mathcal{R} x_{2 n_{k}+1}\right), d\left(\mathcal{R} x_{2 m_{k}}, \mathcal{R} x_{2 n_{k}}\right)\right) .
\end{aligned}
$$

Passing to the limit as $k \rightarrow \infty$, we get

$$
\delta \leq 0+\psi(\delta, 0,0, \delta, \delta)<\delta,
$$

a contradiction. It follows that $\left\{\mathcal{R} x_{n}\right\}$ must be a Cauchy sequence.

Since $\mathcal{X}$ is $(\mathcal{S}, \mathcal{T}, \mathcal{R})$-orbitally complete at $x_{0}$, there exists some $z \in \mathcal{X}$ such that

$$
\mathcal{R} x_{n} \rightarrow z \quad \text { as } n \rightarrow \infty
$$

We will prove that $z$ is a common fixed point of the three mappings $\mathcal{S}, \mathcal{T}$, and $\mathcal{R}$.

We have

$$
\mathcal{R} x_{2 n+1}=\mathcal{S} x_{2 n} \rightarrow z \quad \text { as } n \rightarrow \infty
$$

and

$$
\mathcal{R} x_{2 n+2}=\mathcal{T} x_{2 n+1} \rightarrow z \quad \text { as } n \rightarrow \infty .
$$


Suppose that (a) holds; e.g., let $\mathcal{R}$ be orbitally continuous. Since $\mathcal{S}$ and $\mathcal{R}$ are compatible, we have

$$
\lim _{n \rightarrow \infty} \mathcal{S} \mathcal{R} x_{2 n+2}=\lim _{n \rightarrow \infty} \mathcal{R S} x_{2 n+2}=\mathcal{R} z
$$

From (4.7) and the orbital continuity of $\mathcal{R}$, we have

$$
\mathcal{R}\left(\mathcal{R} x_{n}\right) \rightarrow \mathcal{R} z \quad \text { as } n \rightarrow \infty
$$

Also, $x_{2 n+1} \preceq \mathcal{T} x_{2 n+1}=\mathcal{R} x_{2 n+2}$. Now

$$
\begin{aligned}
d\left(\mathcal{S R} x_{2 n+2}, \mathcal{T} x_{2 n+1}\right) \leq & \psi\left(d\left(\mathcal{R} x_{2 n+1}, \mathcal{R} \mathcal{R} x_{2 n+2}\right), d\left(\mathcal{R} x_{2 n+1}, \mathcal{T} x_{2 n+1}\right)\right. \\
& d\left(\mathcal{R} \mathcal{R} x_{2 n+2}, \mathcal{S} \mathcal{R} x_{2 n+2}\right), d\left(\mathcal{R} x_{2 n+1}, \mathcal{S} \mathcal{R} x_{2 n+2}\right), \\
& \left.d\left(\mathcal{R} \mathcal{R} x_{2 n+2}, \mathcal{T} x_{2 n+1}\right)\right) .
\end{aligned}
$$

Passing to the limit as $n \rightarrow \infty$ in (4.10), using (4.8) and (4.9), and the continuity of $\psi$, if $\mathcal{R} z \neq z$, we obtain

$$
d(\mathcal{R} z, z) \leq \psi(d(\mathcal{R} z, z), 0,0, d(\mathcal{R} z, z), d(\mathcal{R} z, z))<d(\mathcal{R} z, z)
$$

a contradiction, hence

$$
\mathcal{R} z=z
$$

Now, $x_{2 n+1} \preceq \mathcal{T} x_{2 n+1}$ and $\mathcal{T} x_{2 n+1} \rightarrow z$ as $n \rightarrow \infty$, so by the assumption, we have $x_{2 n+1} \preceq z$ and (4.1) gives

$$
\begin{aligned}
d\left(\mathcal{S} z, \mathcal{T} x_{2 n+1}\right) \leq & \psi\left(d\left(\mathcal{R} x_{2 n+1}, \mathcal{R} z\right), d\left(\mathcal{R} x_{2 n+1}, \mathcal{T} x_{2 n+1}\right), d(\mathcal{R} z, \mathcal{S} z)\right. \\
& \left.d\left(\mathcal{R} x_{2 n+1}, \mathcal{S} z\right), d\left(\mathcal{R} z, \mathcal{T} x_{2 n+1}\right)\right)
\end{aligned}
$$

Passing to the limit as $n \rightarrow \infty$ in the above inequality and using (4.11) and the continuity of $\psi$, it follows that if $\mathcal{S} z \neq z$,

$$
d(\mathcal{S} z, z) \leq \psi(0,0, d(\mathcal{S} z, z), d(\mathcal{S} z, z), 0)<d(\mathcal{S} z, z),
$$

which is impossible. Hence,

$$
\mathcal{S} z=z
$$

Now, since $x_{2 n} \preceq \mathcal{S} x_{2 n}$ and $\mathcal{S} x_{2 n} \rightarrow z$ as $n \rightarrow \infty$ implies that $x_{2 n} \preceq z$, from (4.1)

$$
\begin{gathered}
d\left(\mathcal{S} x_{2 n}, \mathcal{T} z\right) \leq \psi\left(d\left(\mathcal{R} z, \mathcal{R} x_{2 n}\right), d(\mathcal{R} z, \mathcal{T} z), d\left(\mathcal{R} x_{2 n}, \mathcal{S} x_{2 n}\right)\right. \\
\left.d\left(\mathcal{R} z, \mathcal{S} x_{2 n}\right), d\left(\mathcal{R} x_{2 n}, \mathcal{T} z\right)\right)
\end{gathered}
$$


Passing to the limit as $n \rightarrow \infty$, and the continuity of $\psi$, we obtain that if $\mathcal{T} z \neq z$,

$$
d(z, \mathcal{T} z) \leq \psi(0, d(z, \mathcal{T} z), 0,0, d(z, \mathcal{T} z))<d(z, \mathcal{T} z)
$$

which gives that

$$
z=\mathcal{T} z
$$

Therefore, $\mathcal{S} z=\mathcal{T} z=\mathcal{R} z=z$, hence $z$ is a common fixed point of $\mathcal{R}, \mathcal{S}$, and $\mathcal{T}$. The proof is similar when $\mathcal{S}$ is orbitally continuous.

Similarly, the result follows when the condition (b) holds.

Now, suppose that the set of common fixed points of $\mathcal{S}, \mathcal{T}$, and $\mathcal{R}$ is totally ordered. We claim that there is a unique common fixed point of $\mathcal{S}, \mathcal{T}$ and $\mathcal{R}$. Assume to the contrary that $\mathcal{S} u=\mathcal{T} u=\mathcal{R} u=u$ and $\mathcal{S} v=\mathcal{T} v=\mathcal{R} v=v$, but $u \neq v$. By supposition, we can replace $x$ by $u$ and $y$ by $v$ in (4.1), and by the lower semi-continuity of $\psi$, we obtain

$$
\begin{aligned}
d(v, u) & =d(\mathcal{T} v, \mathcal{S} u) \leq \psi(d(\mathcal{R} v, \mathcal{R} u), d(\mathcal{R} v, \mathcal{T} v), d(\mathcal{R} u, \mathcal{S} u), d(\mathcal{R} v, \mathcal{S} u), d(u, \mathcal{T} v)) \\
& \leq \psi(d(v, u), 0,0, d(v, u), d(u, v))<d(u, v),
\end{aligned}
$$

a contradiction. Hence, $u=v$. The converse is trivial.

Let $\mathcal{I}$ be the identity mapping on $\mathcal{X}$. Putting $\mathcal{R}=\mathcal{I}$ in Theorem 4 , we obtain easily the following result.

Corollary 3 Let $(\mathcal{X}, d, \preceq)$ be an ordered metric space, and let $\mathcal{T}$ and $\mathcal{S}$ be self-maps on $\mathcal{X}$ satisfying $\psi_{\mathcal{S}}$-contractive conditions for every pair $(x, y) \in \mathcal{O}\left(x_{0} ; \mathcal{S}, \mathcal{T}\right) \times \mathcal{O}\left(x_{0} ; \mathcal{S}, \mathcal{T}\right)($ for some $x_{0}$ ) such that $x$ and $y$ are comparable.

We assume the following hypotheses:

(i) $(\mathcal{S}, \mathcal{T})$ is a.r. at some point $x_{0} \in \mathcal{X}$;

(ii) $\mathcal{X}$ is $(\mathcal{S}, \mathcal{T})$-orbitally complete at $x_{0}$;

(iii) $\mathcal{T}$ and $\mathcal{S}$ are weakly increasing;

(iv) $\mathcal{T}$ and $\mathcal{S}$ are dominating maps;

(v) $\mathcal{S}$ or $\mathcal{T}$ is orbitally continuous at $x_{0}$.

Then $\mathcal{S}$ and $\mathcal{T}$ have a common fixed point. Moreover, the set of common fixed points of $\mathcal{T}$ and $\mathcal{S}$ is totally ordered if and only if $\mathcal{T}$ and $\mathcal{S}$ have one and only one common fixed point.

If $\mathcal{S}=\mathcal{T}$ in Theorem 4, we obtain easily the following consequence.

Corollary 4 Let $(\mathcal{X}, d, \preceq)$ be an ordered metric space, and let $\mathcal{T}$ and $\mathcal{R}$ be self-maps on $\mathcal{X}$ satisfying

$$
d(\mathcal{T} x, \mathcal{T} x) \leq \psi(d(\mathcal{R} x, \mathcal{R} y), d(\mathcal{R} x, \mathcal{T} x), d(\mathcal{R} y, \mathcal{T} y), d(\mathcal{R} x, \mathcal{T} y), d(\mathcal{R} y, \mathcal{T} x))
$$

for every pair $(x, y) \in \mathcal{O}\left(x_{0} ; \mathcal{T}, \mathcal{R}\right) \times \mathcal{O}\left(x_{0} ; \mathcal{T}, \mathcal{R}\right)$ (for some $\left.x_{0}\right)$, where $\psi \in \mathbf{F}$ such that $\mathcal{R} x$ and $\mathcal{R} y$ are comparable.

We assume the following hypotheses: 
(i) $\mathcal{T}$ is a.r. with respect to $\mathcal{R}$ at $x_{0} \in \mathcal{X}$;

(ii) $\mathcal{X}$ is $(\mathcal{T}, \mathcal{R})$-orbitally complete at $x_{0}$;

(iii) $\mathcal{T}$ is weakly increasing with respect to $\mathcal{R}$;

(iv) $\mathcal{T}$ is a dominating map;

(v) $\mathcal{T}$ or $\mathcal{R}$ is orbitally continuous at $x_{0}$.

Then $\mathcal{T}$ and $\mathcal{R}$ have a common fixed point. Moreover, the set of common fixed points of $\mathcal{T}$ and $\mathcal{R}$ is totally ordered if and only if $\mathcal{T}$ and $\mathcal{R}$ have one and only one common fixed point.

Let $\mathcal{I}$ be the identity mapping on $\mathcal{X}$. Putting $\mathcal{R}=\mathcal{I}$ in Corollary 4 , we obtain easily the following consequence.

Corollary 5 Let $(\mathcal{X}, d, \preceq)$ be an ordered metric space, and let $\mathcal{T}$ be a self-map on $\mathcal{X}$ satisfying

$$
d(\mathcal{T} x, \mathcal{T} x) \leq \psi(d(x, y), d(x, \mathcal{T} x), d(y, \mathcal{T} y), d(x, \mathcal{T} y), d(y, \mathcal{T} x))
$$

for every pair $(x, y) \in \mathcal{O}\left(x_{0} ; \mathcal{T}\right) \times \mathcal{O}\left(x_{0} ; \mathcal{T}\right)$ (for some $\left.x_{0}\right)$ such that $x$ and $y$ are comparable.

We assume the following hypotheses:

(i) $\mathcal{T}$ is a.r. at some point $x_{0} \in \mathcal{X}$;

(ii) $\mathcal{X}$ is $\mathcal{T}$-orbitally complete at $x_{0}$;

(iii) $\mathcal{T} x \preceq \mathcal{T}(\mathcal{T} x)$ for all $x \in \mathcal{X}$;

(iv) $\mathcal{T}$ is a dominating map;

(v) $\mathcal{T}$ is orbitally continuous at $x_{0}$.

Then $\mathcal{T}$ has a fixed point. Moreover, the set of fixed points of $\mathcal{T}$ is totally ordered if and only if it is a singleton.

The following example demonstrates the validity of Theorem 4 .

Example 6 Let the set $\mathcal{X}=[0,+\infty)$ be equipped with the usual metric $d$ and the order defined by

$$
x \preceq y \quad \Longleftrightarrow \quad x=y \vee(x, y \in[0,1] \wedge x \geq y) .
$$

Consider the following self-mappings on $\mathcal{X}$ :

$$
\mathcal{R} x=6 x, \quad \mathcal{S} x=\left\{\begin{array}{ll}
\frac{1}{2} x, & 0 \leq x \leq \frac{1}{2}, \\
x, & x>\frac{1}{2},
\end{array} \quad \mathcal{T} x= \begin{cases}\frac{1}{3} x, & 0 \leq x \leq \frac{1}{3}, \\
x, & x>\frac{1}{3} .\end{cases}\right.
$$

Take $x_{0}=\frac{1}{2}$. Then it is easy to show that all the conditions (i)-(iv) and (a)-(b) of Theorem 4 are fulfilled (the condition (iii) on $O\left(x_{0} ; \mathcal{S}, \mathcal{T}, \mathcal{R}\right)$ ). Take $\psi: \mathbb{R}^{+^{5}} \rightarrow \mathbb{R}^{+}$given by

$$
\psi\left(t_{1}, t_{2}, t_{3}, t_{4}, t_{5}\right)=\frac{1}{6} \max \left\{t_{1}, t_{2}, t_{3}, \frac{t_{4}}{2}, \frac{t_{5}}{2}\right\},
$$

and $\psi \in \mathbf{F}$. Then the contractive condition (4.1) takes the form

$$
\left|\frac{1}{2} x-\frac{1}{3} y\right| \leq \frac{1}{6} \max \left\{|6 x-6 y|, \frac{11}{2} x, \frac{17}{3} y, \frac{1}{2}\left|6 x-\frac{1}{3} y\right|, \frac{1}{2}\left|6 y-\frac{1}{2} x\right|\right\},
$$


for $x, y \in O\left(x_{0} ; \mathcal{S}, \mathcal{T}, \mathcal{R}\right)$. Using substitution $y=t x, t \geq 0$, the last inequality reduces to

$$
|3-2 t| \leq \max \left\{6|1-t|, \frac{11}{2}, \frac{17}{3} t, \frac{1}{2}\left|6-\frac{1}{3} t\right|, \frac{1}{2}\left|6 t-\frac{1}{2}\right|\right\},
$$

and can be checked by discussion on possible values for $t \geq 0$. Hence, all the conditions of Theorem 4 are satisfied and $\mathcal{S}, \mathcal{T}, \mathcal{R}$ have a common fixed point (which is 0 ).

\section{Existence of a common solution of integral equations}

Consider the system of integral equations:

$$
\begin{cases}u(t)=\int_{0}^{T} K_{1}(t, s, u(s)) d s, & t \in[0, T] \\ u(t)=\int_{0}^{T} K_{2}(t, s, u(s)) d s, & t \in[0, T]\end{cases}
$$

where $T>0$ and $K_{1}, K_{2}:[0 ; T] \times[0 ; T] \times \mathbb{R} \rightarrow \mathbb{R}$ are continuous functions. The purpose of this section is to give an existence theorem for a solution of (5.1).

We consider the set $C(I, \mathbb{R})(I=[0, T])$ of real continuous functions defined on $I$. This set with the metric given by

$$
d(u, v)=\max _{t \in I}|u(t)-v(t)|, \quad \forall u, v \in C(I, \mathbb{R})
$$

is a complete metric space. $C(I, \mathbb{R})$ can also be equipped with the partial order $\preceq$ given by

$$
u, v \in C(I, \mathbb{R}), \quad u \preceq v \quad \Leftrightarrow \quad u(t) \leq v(t), \quad \forall t \in I .
$$

Moreover, in [7] it is proved that $(C(I, \mathbb{R}), d, \preceq)$ is regular.

Consider the mappings $\mathcal{T}, \mathcal{S}: C(I, \mathbb{R}) \rightarrow C(I, \mathbb{R})$ defined by

$$
\begin{aligned}
& \mathcal{T} u(t)=\int_{0}^{T} K_{1}(t, s, u(s)) d s, \quad \text { for all } C(I, \mathbb{R}), t \in I, \\
& \mathcal{S} u(t)=\int_{0}^{T} K_{2}(t, s, u(s)) d s, \quad \text { for all } C(I, \mathbb{R}), t \in I .
\end{aligned}
$$

Clearly, $u$ is a solution of (5.1) if and only if it is a common fixed point of $\mathcal{T}$ and $\mathcal{S}$.

We shall prove the existence of a common fixed point of $\mathcal{T}$ and $\mathcal{S}$ under certain conditions.

Theorem 5 Suppose that the following hypotheses hold:

(H1) for all $t, s \in I, u \in C(I, \mathbb{R})$, we have

$$
K_{1}(t, s, u(s)) \leq K_{2}\left(t, s, \int_{0}^{T} K_{1}(s, \tau, u(\tau)) d \tau\right)
$$

(H2) for all $t, s \in I, u \in C(I, \mathbb{R})$, we have

$$
K_{2}(t, s, u(s)) \leq K_{1}\left(t, s, \int_{0}^{T} K_{2}(s, \tau, u(\tau)) d \tau\right)
$$


(H3) there exist a continuous function $\alpha: I \times I \rightarrow \mathbb{R}^{+}$and $\psi \in \mathbf{F}$ such that

$$
\begin{aligned}
& \left|K_{1}(t, s, x(s))-K_{2}(t, s, y(s))\right| \\
& \quad \leq \alpha(t, s) \psi(d(x, y), d(x, \mathcal{T} x), d(y, \mathcal{S} y), d(x, \mathcal{S} y), d(y, \mathcal{T} x))
\end{aligned}
$$

for all $t, s \in I$ and $x, y \in C(I, \mathbb{R})$ such that $x \succeq y$;

(H4) $\sup _{t \in I} \int_{0}^{T} \alpha(t, s) d s \leq 1$.

Then the system of integral equations (5.1) has a solution $u^{*} \in C(I, \mathbb{R})$.

Proof Let $u \in C(I, \mathbb{R})$. Using (H1), for all $t \in I$, we have

$$
\begin{aligned}
\mathcal{T} u(t) & =\int_{0}^{T} K_{1}(t, s, u(s)) d s \\
& \leq \int_{0}^{T} K_{2}\left(t, s, \int_{0}^{T} K_{1}(s, \tau, u(\tau)) d \tau\right) d s \\
& =\int_{0}^{T} K_{2}(t, s, \mathcal{T} u(s)) d s \\
& =\mathcal{S} \mathcal{T} u(t) .
\end{aligned}
$$

Similarly, using (H2), for all $t \in I$, we have

$$
\begin{aligned}
\mathcal{S} u(t) & =\int_{0}^{T} K_{2}(t, s, u(s)) d s \\
& \leq \int_{0}^{T} K_{1}\left(t, s, \int_{0}^{T} K_{2}(s, \tau, u(\tau)) d \tau\right) d s \\
& =\int_{0}^{T} K_{1}(t, s, \mathcal{S} u(s)) d s \\
& =\mathcal{T} \mathcal{S} u(t) .
\end{aligned}
$$

Then we have $\mathcal{T} u \preceq \mathcal{S} \mathcal{T} u$ and $\mathcal{S} u \preceq \mathcal{T S} u$ for all $u \in C(I, \mathbb{R})$. This implies that $\mathcal{T}$ and $\mathcal{S}$ are weakly increasing.

Now, for all $u, v \in C(I, \mathbb{R})$ such that $v \preceq u$, by (H3) and (H4), we have

$$
\begin{aligned}
& |\mathcal{T} u(t)-\mathcal{S} v(t)| \\
& \quad \leq \int_{0}^{T}\left|K_{1}(t, s, u(s))-K_{2}(t, s, v(s))\right| d s \\
& \quad \leq \int_{0}^{T} \alpha(t, s) \psi(d(u, v), d(u, \mathcal{T} u), d(v, \mathcal{S} v), d(u, \mathcal{S} v), d(v, \mathcal{T} u)) d s \\
& \quad=\left(\int_{0}^{T} \alpha(t, s) d s\right) \psi(d(u, v), d(u, \mathcal{T} u), d(v, \mathcal{S} v), d(u, \mathcal{S} v), d(v, \mathcal{T} u)) \\
& \quad \leq \psi(d(u, v), d(u, \mathcal{T} u), d(v, \mathcal{S} v), d(u, \mathcal{S} v), d(v, \mathcal{T} u)) .
\end{aligned}
$$

Hence, we have proved that for all $u, v \in C(I, \mathbb{R})$ such that $u \succeq v$, we have

$$
d(\mathcal{T} u, \mathcal{S} v) \leq \psi(d(u, v), d(u, \mathcal{T} u), d(v, \mathcal{S} v), d(u, \mathcal{S} v), d(v, \mathcal{T} u))
$$


Now, all the hypotheses of Corollary 1 are satisfied. Then $\mathcal{T}$ and $\mathcal{S}$ have a common fixed point $u^{*} \in C(I, \mathbb{R})$, that is, $u^{*}$ is a solution of the system (5.1).

\section{Competing interests}

The authors declare that they have no competing interests.

\section{Authors' contributions}

All authors contributed equally and significantly in writing this paper. All authors read and approved the final manuscript.

\section{Author details}

'Department of Mathematics, Disha Institute of Management and Technology, Satya Vihar, Vidhansabha-Chandrakhuri Marg, Mandir Hasaud, Raipur, Chhattisgarh 492101, India. ${ }^{2}$ Faculty of Mechanical Engineering, University of Belgrade, Kraljice Marije 16, Beograd, 11120, Serbia. ${ }^{3}$ Faculty of Mathematics, University of Belgrade, Studentski trg 16, Beograd, 11000 , Serbia.

\section{Acknowledgements}

The authors thank the referees for their valuable comments that helped them to improve the text. The second and third authors are thankful to the Ministry of Science and Technological Development of Serbia.

\section{Received: 19 August 2012 Accepted: 25 October 2012 Published: 13 November 2012}

\section{References}

1. Banach, S: Sur les opérations dans les ensembles abstraits et leur application aux equations itegrales. Fundam. Math. 3, 133-181 (1922)

2. Nashine, HK: New fixed point theorems for mappings satisfying generalized weakly contractive condition with weaker control functions. Ann. Pol. Math. 104, 109-119 (2012)

3. Browder, FE, Petryshyn, WV: Construction of fixed points of nonlinear mappings in Hilbert spaces. J. Math. Anal. Appl. 20, 197-228 (1967)

4. Ćirić, LB: A generalization of Banach's contraction principle. Proc. Am. Math. Soc. 45, 267-273 (1974)

5. Sastry, KPR, Naidu, SVR, Rao, IHN, Rao, KPR: Common fixed points for asymptotically regular mappings. Indian J. Pure Appl. Math. 15, 849-854 (1984)

6. Ran, ACM, Reurings, MCB: A fixed point theorem in partially ordered sets and some applications to matrix equations. Proc. Am. Math. Soc. 132, 1435-1443 (2004)

7. Nieto, JJ, López, RR: Contractive mapping theorems in partially ordered sets and applications to ordinary differential equations. Order 22, 223-239 (2005)

8. Golubović, Z, Kadelburg, Z, Radenović, S: Common fixed points of ordered g-quasicontractions and weak contractions in ordered metric spaces. Fixed Point Theory Appl. 2012, 20 (2012). doi:10.1186/1687-1812-2012-20

9. Nashine, HK, Altun, l: Fixed point theorems for generalized weakly contractive condition in ordered metric spaces. Fixed Point Theory Appl. 2011, Article ID 132367 (2011)

10. Nashine, HK, Altun, I: A common fixed point theorem on ordered metric spaces. Bull. Iran. Math. Soc. (to appear)

11. Nashine, HK, Samet, B: Fixed point results for mappings satisfying $(\psi, \varphi)$-weakly contractive condition in partially ordered metric spaces. Nonlinear Anal. 74, 2201-2209 (2011)

12. Nashine, HK, Samet, B, Vetro, C: Monotone generalized nonlinear contractions and fixed point theorems in ordered metric spaces. Math. Comput. Model. 54, 712-720 (2011)

13. Chen, C-M: Fixed point theorems for $\psi$-contractive mappings in ordered metric spaces. J. Appl. Math. 2012, Article ID 756453 (2012)

14. Harjani, J, López, B, Sadarangani, K: Fixed point theorems for weakly C-contractive mappings in ordered metric spaces. Comput. Math. Appl. 61, 790-796 (2011)

15. Dhage, BC: Condensing mappings and applications to existence theorems for common solution of differential equations. Bull. Korean Math. Soc. 36, 565-578 (1999)

16. Dhage, BC, O'Regan, D, Agarwal, RP: Common fixed point theorems for a pair of countably condensing mappings in ordered Banach spaces. J. Appl. Math. Stoch. Anal. 16, 243-248 (2003)

17. Vetro, CL: Common fixed points in ordered Banach spaces. Matematiche $63,93-100$ (2008)

18. Nashine, HK, Samet, B, Vetro, C: Common fixed points of strictly weakly isotone increasing mappings in ordered metric spaces. Filomat (submitted)

19. Abbas, M, Nazir, T, Radenović, S: Common fixed point of four maps in partially ordered metric spaces. Appl. Math. Lett. 24, 1520-1526 (2011)

20. Altun, I, Simsek, H: Some fixed point theorems on ordered metric spaces and application. Fixed Point Theory Appl. 2010, Article ID 621492 (2010)

21. Jungck, G: Commuting mappings and fixed points. Am. Math. Mon. 83, 261-263 (1976)

22. Jungck, G: Compatible mappings and common fixed points. Int. J. Math. Math. Sci. 9, 771-779 (1986)

doi:10.1186/1687-1812-2012-203

Cite this article as: Nashine et al.: Modified $\psi$-contractive mappings in ordered metric spaces and applications. Fixed Point Theory and Applications 2012 2012:203. 